Design of a Rayleigh-Taylor experiment to Measure Strength at High Pressures

K. O. Mikaelian

May 24, 2010

Physics of Plasmas 
This document was prepared as an account of work sponsored by an agency of the United States government. Neither the United States government nor Lawrence Livermore National Security, LLC, nor any of their employees makes any warranty, expressed or implied, or assumes any legal liability or responsibility for the accuracy, completeness, or usefulness of any information, apparatus, product, or process disclosed, or represents that its use would not infringe privately owned rights. Reference herein to any specific commercial product, process, or service by trade name, trademark, manufacturer, or otherwise does not necessarily constitute or imply its endorsement, recommendation, or favoring by the United States government or Lawrence Livermore National Security, LLC. The views and opinions of authors expressed herein do not necessarily state or reflect those of the United States government or Lawrence Livermore National Security, LLC, and shall not be used for advertising or product endorsement purposes. 


\title{
Design of a Rayleigh-Taylor experiment to measure strength at high pressures
}

\author{
Karnig O. Mikaelian
}

Lawrence Livermore National Laboratory, Livermore, California 94551

We present a design to measure the strength of a metal at very high pressures using the Rayleigh-Taylor instability. The target consists of a metal foil behind a tamper and an ablator driven by soft x-rays generated in a hohlraum at the Nation Ignition Facility (NIF) or Laser MegaJoule (LMJ). Since ignition capsules and strength targets both call for quasiadiabatic drives, we use the early, 0-16 ns, part of the ignition pulse to drive an almost-10-Mb strength experiment. We also discuss variations on how initial perturbations may be placed at the metal/tamper interface, resulting in a high-pressure microindentation technique. We illustrate the time-evolution of perturbations under various assumptions concerning tantalum strength.

PACS numbers 62.20.F-, 62.50.-p, 47.20.Ma

\section{INTRODUCTION}

Mechanical properties of materials such as compressibility, strength, viscosity, etc., are important for industrial applications, planetary and geophysical explorations, as well as the theoretical understanding of plasticity from the atomic scale (dislocation dynamics) to the mesoscopic scale (sliding of grains) ${ }^{1-4}$. These properties are particularly difficult and challenging to obtain at high pressures; static techniques, such as diamond anvil 
cells, have a theroretical limit ${ }^{5}$ of about $5 \mathrm{Mb}$ and are often conducted ${ }^{6}$ in the $1 \mathrm{Mb}$ range. Much higher pressures can, in principle, be achieved using dynamic techniques and the method proposed in this paper falls in this category. Such techniques are, naturally, more demanding as one must compress the sample and simultaneously interrogate its properties at high pressures.

Dynamic experiments have been successfully carried out at the $\mathrm{Z}$ and Omega facilities: Tantalum, molybdenum, copper and beryllium have been studied at the Zfacility using magnetic compression ${ }^{7,8}$. At the Omega laser facility diamond ${ }^{9,10}$ and, more recently, vanadium ${ }^{11}$ has been used to study equation-of-state and strength properties. Isentropic compression on both the $\mathrm{Z}$ and Omega facilities has been used ${ }^{7-11}$ to compress the sample without melting it. The method and calculations presented in this paper aim for higher pressures, about $10 \mathrm{Mb}$, are intended for the NIF laser ${ }^{12,13}$, and are also based on isentropic compression of a sample without melting it. We use tantalum for illustration.

As discussed in Ref. 13, ramp compression experiments are under consideration for NIF to study material properties. In those experiments a short laser pulse shocks and energizes a reservoir that subsequently unloads across a gap and ramp-compresses the target. ${ }^{14}$ In this aspect it resembles the technique used first by Barnes et al. ${ }^{15}$ with high explosives as the reservoir. In our design the x-rays are absorbed by a high-Z-doped ablator and the laser pulse is shaped temporally to drive a series of shocks into the sample, again having perturbations whose growth is a measure of strength.

This research was undertaken to correlate and cross-fertilize two completely separate platforms: Ignition experiments and materials experiments. It is well-known ${ }^{17-19}$ that ignition requires an adiabatic implosion, achieved via pulse-shaping, to form a very high 
density core without preheat. Similarly, materials experiments require a quasi-isentropic drive to push the sample without heating or melting it. The question emerges naturally: Can one use the ignition pulse to drive a materials experiment? The answer, we believe, is yes we can.

A design for measuring the high-pressure yield strength of tantalum is presented in Sec. II. Variations with the primary aim of simplifying target fabrication are presented in Sec. III. Conclusions are given in Sec. IV.

\section{DESIGN OF EXPERIMENT}

Fig. 1 shows the ignition pulse expected to be used to implode cryogenic DT capsules in a hohlraum on NIF. ${ }^{12,13,17,18,20}$ We use exactly the same pulse until about 16 ns at which time the laser is turned off. In this sense we piggyback the ignition experiments capitalizing on their experience to generate the required pulse, not taxing the laser system at all by stopping well short of the peak: $140 \mathrm{eV}$ versus $300 \mathrm{eV}$. We believe this selfimposed constraint benefits both efforts, although it makes the RT target design more challenging.

Another constraint we have adopted is to use "standard" materials for ablator and tamper. The design is shown in the inset of Fig. 1: The ablator is $75 \mu \mathrm{m}$ of brominated polystyrene, $\mathrm{CHBr}$, with $12.5 \%$ atomic fraction of bromine, followed by $25 \mu \mathrm{m}$ of $\mathrm{CH}$, then the metal foil, Ta, also $25 \mu \mathrm{m}$ thick, and finally $200 \mu \mathrm{m}$ of $\mathrm{CH}$. More exotic ablators (diamond, beryllium,...) have been used in the past but we believe the simplicity of the design shown in Fig. 1, based on more commonly used materials, will be advantageous in fabricating the target. 
We can drive the target by the truncated temperature source shown in Fig. 1 or, better yet, by the frequency-dependent-source (FDS) that gave rise to that Tr. The advantage of the FDS is that it is more realistic, containing the gold $\mathrm{M}$ and $\mathrm{L}$ lines that are more penetrating than the $400-500 \mathrm{eV}$ photons of the main $140-\mathrm{eV}$ temperature source. In fact, the bromine was added in the ablator to shield the metal foil from gold preheat (This is standard practice, sometimes germanium replacing bromine). We found the $12.5 \%$ of bromine more than sufficient to absorb preheat.

Of course x-rays are not the only source of the unwanted heating of the foil - The hydrodynamic push, generated by several mini-shocks as the laser is turned on, must not heat and melt the foil and thus lose all information about strength. In short, the target must move in a quasi-isentropic fashion, as discussed in the Introduction. This was necessarily the main criterion that determined the choice of the target shown in Fig. 1. Although melt cannot be avoided throughout the whole foil at all times, we found that the foil remains solid long enough to affect the growth of perturbations at the $\mathrm{CH} / \mathrm{Ta}$ upper interface.

Before we study the evolution of perturbations in two-dimensions (2D), we make sure that the one-dimensional (1D) motion of the foil is sufficiently isentropic. Using the hydrodynamic code CALE with multigroup (55) diffusion for radiation, ${ }^{21}$ we display in Fig. 2 the 1D properties of the tantalum near its upper surface where 2D perturbations will later be placed. We show the pressure, density, velocity, electron temperature and melt temperature, all as functions of time. The peak pressure reaches almost $10 \mathrm{Mb}$, the density more than doubles, the peak speed of the interface is about $5 \mu \mathrm{m} / \mathrm{ns}$, and the temperature remains below $0.5 \mathrm{eV}$, well below the melt temperature of $2 \mathrm{eV}$. These are ideal, 1D properties required for the design to qualify as a viable candidate for RT 
experiments. For example, had the target melted in 1D there would be no point in performing time-consuming 2D calculations because strength effects would be absent. In other words these are necessary, but by no means sufficient conditions a design must meet.

Having passed these preliminary requirements we proceed to assess the sensitivity of the design to strength. We impose $2 \mathrm{D}$ sinusoidal perturbations at the upper $\mathrm{CH} / \mathrm{Ta}$ interface. $\mathrm{CALE}^{21}$ is a $2 \mathrm{D}$ hydrocode in planar, cylindrical, or spherical geometry. We use planar geometry for ease of diagnostics, assumed to be backlit radiography as, for example, in Ref. 11.

Both the wavelength $\lambda$ and the amplitude $\eta_{0}$ that define the curve $\eta_{0} \sin (2 \pi x / \lambda)$ influence the RT instability, particularly in solids. The $\lambda$-effect was first pointed out in the classic work of Miles, ${ }^{22}$ and the $\eta_{0}$-effect was predicted by Drucker. ${ }^{23}$ More modern analytic formulations confirm the influence of both $\lambda$ and $\eta_{0}$ (see, for example, Refs. 2426). Although details vary among the analyses, they all agree that small $\eta_{0}$ 's and shorter $\lambda$ 's are more sensitive to strength effects. ${ }^{22-26}$ We base our predictions on $2 \mathrm{D}$ direct numerical simulations, taking into account the third and perhaps most severe constraint of experimental diagnostics.

The requirements of diagnostics run exactly opposite to those of sensitivity to strength, making it difficult to satisfy both. Diagnostics require large amplitudes and long wavelengths to well resolve the experimental radiograph, while strength is most sensitive to small amplitudes and short wavelengths. After a series of calculations we found a wavelength, specifically $25 \mu \mathrm{m}$, and amplitude, specifically $1 \mu \mathrm{m}$, that can be diagnosed and at the same time is sensitive to strength. We ran several calculations at different wavelengths and amplitudes before settling on this choice, and they all verified the above 
discussed dualism: Larger wavelength and/or amplitudes are better diagnosed but are not sensitive to strength, and conversely shorter wavelengths and/or amplitudes are better probes of strength, but of course difficult to detect.

Fig. 3 displays our results, the time-evolution of the peak-to-valley amplitude, initially equal to $2 \eta_{0}=2 \mu \mathrm{m}$, under four different assumptions for Ta strength. Case $\mathrm{A}$ is the fluid or no-strength case, growing $\sim 8.5$ times to $17 \mu \mathrm{m}$. Case B assumes SteinbergGuinan $^{27}$ (SG) strength for Ta. Case C assumes Steinberg-Lund ${ }^{28}(\mathrm{SL})$, and case D is a variation of SG with double its pressure-hardening coefficient A. The SG model is described briefly in the Appendix - It is probably the most commonly used model for strength at high pressures, though there are many other new models as this remains an active field of research ${ }^{29}$.

The largest contrast between a strength and no-strength case in Fig. 3 is about 1.7. If Ta turns out to have even higher strength then this contrast will increase. Conversely, a weaker Ta will behave like a fluid. We repeat that the contrast, within a given model, increases if we impose shorter $\lambda$ 's or smaller $\eta$ 's, but we believe those will be almost unobservable experimentally.

It does not follow that one should not experiment with other $\lambda$ 's, particularly long ones. In fact, the lack of sensitivity at long $\lambda$ can serve as a check of the simulation because a fairly unique, meaning independent of the strength model, prediction could be tested experimentally. For $\lambda=50 \mu \mathrm{m}$ and $\eta_{0}=2 \mu \mathrm{m}$ we found the peak-to-valley increasing from $4 \mu \mathrm{m}$ to $18 \mu \mathrm{m}$ in the fluid case, while turning on the SG strength model made only a $5 \%$ difference, compared with the $30 \%$ difference shown in Fig. 3 for the $\lambda=25 \mu \mathrm{m}$, $\eta_{0}=1 \mu \mathrm{m}$ case. Fig. 4 shows snapshots for this latter case, with the pseudocolor field 
displaying the yield strength in the tantalum at various times. Note that at $17 \mathrm{~ns}$ certain parts of the metal have melted while other parts maintain their strength with a maximum of about $90 \mathrm{~kb}$ (red), which is more than ten times the initial strength of $7.7 \mathrm{~kb}$. This increase is due primarily (but not exclusively) to pressure hardening controlled by the Aterm - See the Appendix.

\section{VARIATIONS}

All the 2D simulations we have discussed so far start with a sinusoidal perturbation at the $\mathrm{CH} / \mathrm{Ta}$ interface, a natural configuration lending itself to analytic treatment, ${ }^{23-26}$ implying that both the metal and the tamper are conformally machined or pressed together so as to form one sinusoidally-shaped interface between them, as shown in Fig. 5a. We believe all previous treatments, be they analytic, numerical or experimental, have started with an interface as shown in Fig. 5a. However, from a numerical and particularly experimental standpoint this is not a requirement - At least two other configurations, shown as Figs. 5b and 5c, are equally, if not more, viable. In Fig. 5b only the metal is machined or coined into a sinusoidal shape and the tamper is flat, while in Fig. 5c the metal is flat and only the tamper is shaped. Actually the shape also does not have to be sinusoidal if one is not doing an analytic eigenvalue assessment, but we found no big advantage in switching to other shapes.

Our simulations starting with the conditions shown in Fig. $5 \mathrm{~b}$ and $5 \mathrm{c}$ indicate that they both provide as good an experimental signal as Fig. 5a. However, we see no particular advantage in Fig. 5b and will not discuss it any further. Fig 5c or Method C, on the other hand, does have the experimental advantage that the metal does not have to be machined. Machining or coining into a particular shape could alter the strength properties 
one is trying to measure. Experimentally, Method C is also the more "natural" configuration to test the strength of a material as it is ordinarily put to use. This method is inspired by the well-known micro- or nano-indentation techniques used widely at ambient pressures - See, for example, Refs. 30-32. It is particularly useful when experimenting with crystalline materials that come with flat surfaces and where machining the surface of the sample may be damaging or may prove too difficult or too invasive, and the method of Fig. 5c would be a welcome simplification.

The same principles (Sec. II) that governed our choice of $\lambda$ and of $\eta_{0}$ for Method A also lead us to a fairly unique choice in the case of Method $\mathrm{C}$ : The wavelength and the final amplitude must be large enough to be experimentally observable, yet small enough to differentiate between a "strong" and "weak" material. We found $\lambda=25 \mu \mathrm{m}$ to again be a good choice, and $\eta_{0}=2 \mu \mathrm{m}$ large enough to yield an observable signal, yet not too large as to mask the effect of strength. This is another advantage of Method C: Deep grooves, 4 $\mu \mathrm{m}$ peak-to-valley, can be well defined and machined into the $\mathrm{CH}$ and dominate over the small-scale irregularities that are almost always present. These grooves act as a "hammer" that pushes into the sample and just how much the sample deforms is a measure of its strength or lack of it. As usual, in taking a late time radiograph with high energy $x$-rays one observes only the peaks and valleys of the metal sample itself $(Z=73$ for Ta) while the $\mathrm{CH}$ and $\mathrm{CHBr}$ remain transparent $(\mathrm{Z}=3.5$ and 7.8$)$.

Fig. 6 shows the peak-to-valley amplitudes for this case under the same four assumptions as in Fig. 3 concerning Ta strength. It grows from zero (initially flat Ta) to a maximum of about $8 \mu \mathrm{m}$ in the no-strength case, and to a minimum of about $2 \mu \mathrm{m}$ in the modified SG, the strongest case. Snapshots of the standard SG case are shown in Fig. 7 with the colors again indicating yield strength on the same scale as in Fig. 4. As expected, 
the sinusoidal groove in the $\mathrm{CH}$ has inverted upon the passage of the first shock and acts as a "hammer" pushing into the metal. In contrast with Fig. 4, the metal still has some strength, about $18 \mathrm{~kb}$ (blue) left in its backside at $20 \mathrm{~ns}$.

In Fig. 6 we see a difference of about $80 \%$ between the SG and the no-strength case, compared with about $30 \%$ for the Method A shown in Fig. 3. This suggests that we can "afford" to go to larger wavelengths and still maintain sensitivity to strength. Indeed, a $\lambda=50 \mu \mathrm{m}, \eta_{0}=3 \mu \mathrm{m}$ simulation with Method C produced approximately $28 \%$ difference between the SG and the no-strength cases, growing from 0 to $3.9 \mu \mathrm{m}$ and to $5 \mu \mathrm{m}$, respectively, which we believe can be easily diagnosed.

\section{CONCLUDING REMARKS}

We have presented a design that uses the early part of an ignition pulse (Fig. 1) to push and indent a tantalum sample, with the amount of indentation measuring the strength of the sample, as performed originally by Barnes et al. with HE products pushing an aluminum sample. ${ }^{15}$ We searched through a large design space varying ablator, tamper, and metal thicknesses, as well as $\lambda$ and $\eta_{0}$, and have presented our best experimental candidate that can be diagnosed and yet has good contrast, meaning sensitivity to the strength of the metal. We believe the design can be further improved by indulging into the additional freedom of varying the drive. Even within the constraints of using the ignition pulse as is, we believe such experiments are viable and informative without taxing the laser system. In addition to $\mathrm{NIF}^{12,13,20}$ one may consider other systems such as the $\mathrm{LMJ}^{33}$ as a possible platform. The laser pulse will probably vary from what we have used but we are confident that any quasi-isentropic ignition pulse can do doubleduty as a driver for material properties of solids. 
Given a strength model the design seeks to maximize the contrast between the nostrength and strength cases. Since viscous effects are not included once a zone melts or yields, we expect that contrast to be even larger. Of course the perturbations cannot grow any faster than the no-strength case; however, it is possible for them not to grow at all if the strength turns out to be much higher. Conversely, if the strength is less than SG there may be little or no contrast.

It is interesting that Method C (Fig. 5c) has better contrast, probably reflecting the well-known fact that smaller $\eta_{0}$ 's lead to smaller growth factors in strong materials ${ }^{23,34}$ It is the ultimate "small $\eta_{0}$ " experiment with $\eta_{0}=0$ on the surface of the metal. We have assumed that it is desirable to produce an observable peak-to-valley variation, and not be content with a small or no variation at all (flat foil accelerating and remaining flat). To produce a sizable variation one must use a large enough "hammer", meaning perturbations in the $\mathrm{CH}$ tamper. Yet it must not be so large as to wipe out the strength/nostrength contrast.

Methods $\mathrm{A}$ and $\mathrm{C}$ are compared graphically in Fig. 8 with the strength and nostrength cases displayed side by side for each method. Although Method A leads to larger over all growth, Method $\mathrm{C}$ has better contrast. Add the experimental advantage that one does not have to prepare a metal surface machined with specific perturbations (which may affect the properties of the sample), and we believe it is more advantageous. Method C also leads naturally to the concept that one may use the same "hammer" to drive other metals, especially if they have approximately the same density as the original one. For example, 18 carat gold ( $75 \% \mathrm{Au}, 25 \% \mathrm{Cu}$ by volume) has a density very close to that of tantalum, $16.67 \mathrm{~g} / \mathrm{cm}^{3}$. 
We hope the calculations, techniques, and ideas presented in this work will prove useful in future experiments.

\section{ACKNOWLEDGMENTS}

I am grateful to Dan Clark for providing the ignition pulse, a photon-frequency dependent source. This work was performed under the auspices of the U. S. Department of Energy by Lawrence Livermore National Laboratory under Contract DE-AC52-07NA27344.

\section{APPENDIX: THE STEINBERG-GUINAN MODEL}

The yield strength $Y$ of a metal in the Steinberg-Guinan model ${ }^{27}$ is given by

$$
Y=Y_{0}\left[1+\beta\left(\varepsilon_{0}+\varepsilon_{p}\right)\right]^{n}\left\{1+A P\left(\rho_{0} / \rho\right)^{1 / 3}-B(T-300)\right\} .
$$

$Y_{0}, \varepsilon_{0}$, and $\rho_{0}$ are the initial (zero-pressure) yield strength, strain, and density, respectively, while $\varepsilon_{p}$ is the equivalent plastic strain. $A$ and $B$ are the coefficients of the pressure-hardening and thermal-softening terms, respectively. $T$ is the temperature in Kelvin, and $P$ is pressure. The coefficient $Y_{0}\left[1+\beta\left(\varepsilon_{0}+\varepsilon_{p}\right)\right]^{n}$ is limited by maximum work hardening,

$$
Y_{0}\left[1+\beta\left(\varepsilon_{0}+\varepsilon_{p}\right)\right]^{n} \leq Y_{w h m x} .
$$


The code calculates $P, \rho, T$, and $\varepsilon_{p}$ as functions of time and position in the metal. For tantalum, the recommended values for the constants $\operatorname{are}^{27}: Y_{0}=7.7 \mathrm{~kb}, \beta=22, \varepsilon_{0}=0$, $n=0.283, A=1.45 / \mathrm{Mb}, \rho_{0}=16.69 \mathrm{~g} / \mathrm{cm}^{3}, B=1.3 \times 10^{-4} / \mathrm{K}$, and $Y_{w h m x}=11 \mathrm{~kb}$.

For the "modified SG" curves D in Figs. 3 and 6 we replaced $A$ by $2 A$, resulting in "stronger" tantalum and consequently less growth. 


\section{REFERENCES}

${ }^{1}$ T. H. Courtney, Mechanical Behavior of Materials, McGraw Hill, Boston, 2000.

${ }^{2}$ J. Hodowany, G. Ravichandran, A. J. Rosakis, and P. Rosakis, Exp. Mech. 40, 113 (2000).

${ }^{3}$ O. Grosset, C. Sohn, and F. Deschamps, Planet. Space Sci. 48, 617 (2000).

${ }^{4}$ B. B. Karki, L. Stixrude, and R. M. Wentzcovitch, Rev. Geophys. 39, 507 (2001).

${ }^{5}$ S. T. Weir, J. Akella, and C. Aracne-Ruddle, Appl. Phys. Lett. 77, 3400 (2000).

${ }^{6}$ A. Dewaele and P. Lonbeure, Phys. Rev. B 72, 134106 (2005).

${ }^{7}$ D. B. Reisman, A. Toor, R. C. Cauble, C. A. Hall, J. R. Asay, M. D. Knudson, and M. D. Furnish, J. Appl. Phys. 89, 1625 (2001).

${ }^{8}$ J. R. Asay, T. Ao, T. J. Vogler, J-P. Davis, and G. T. Gray, J. Appl. Phys. 106, 073515 (2009).

${ }^{9}$ D. K. Bradley, J. H. Eggert, R. F. Smith, S. T. Prisbrey, D. G. Braun, J. Biener, A. V. Hamza, R. E. Rudd, and G. W. Collins, Phys. Rev. Lett. 102, 075503 (2009).

${ }^{10}$ R. S. McWilliams, J. H. Eggert, D. G. Hicks, D. K. Bradley, P. M. Celliers, D. K. Spaulding, T. R. Boehly, G. W. Collins, and R. Jeanloz, Phys. Rev. B 81, 014111 (2010).

${ }^{11}$ H-S. Park, K. T. Lorenz, R. M. Cavallo, S. M. Pollaine, S. T. Prisbrey, R. E. Rudd, R. C. Becker, J. V. Bernier, and B. A. Remington, Phys. Rev. Lett. 104, 135504 (2010); HS. Park, B. A. Remington, R. C. Becker, J. V. Bernier, R. M. Cavallo, K. T. Lorenz, S. M. Pollaine, S. T. Prisbrey, R. E. Rudd, and N. R. Barton, Phys. Plasmas 17, 056314 (2010). 
${ }^{12}$ J. D. Lindl, P. Amendt, R. L. Berger, S. G. Glendinning, S. H. Glenzer, S. W. Haan, R. L. Kauffman, O. L. Landen, and L. J. Suter, Phys. Plasmas 11, 339 (2004).

${ }^{13}$ E. I. Moses, R. N. Boyd, B. A. Remington, C. J. Keane, and R. Al-Ayat, Phys. Plasmas 16, 041006 (2009).

${ }^{14}$ J. Edwards, K. T. Lorenz, B. A. Remington, S. Pollaine, J. Colvin, D. Braun, B. F. Lasinski, D. Reisman, J. M. McNaney, J. A. Greenough, R. Wallace, H. Louis, and D. Kalantar, Phys. Rev. Lett. 92, 075002 (2004).

${ }^{15}$ J. F. Barnes, P. J. Blewett, R. G. McQueen, and K. A. Meyer, J. Appl. Phys. 45, 727 (1974).

${ }^{16}$ Lord Rayleigh, Scientific Papers, 2, ( Dover, New York, 1965); G. I. Taylor, Proc. R. Soc. London Ser. A 201, 192 (1950).

${ }^{17}$ J. D. Lindl, Inertial Confinement Fusion (Springer, New York, 1998).

${ }^{18}$ W. J. Krauser, N. M. Hoffman, D. C. Wilson, B. H. Wilde, W. S. Varnum, D. B. Harris, F. J. Swenson, P. A. Bradley, S. W. Haan, S. M. Pollaine, A. S. Wan, J. C. Moreno, and P. A. Amendt, Phys. Plas. 3, 2084 (1996).

${ }^{19}$ S. Skupsky, J. A. Marozas, R. S. Craxton, R. Betti, T. J. B. Collins, J. A. Delettrez, V. N. Goncharov, P. W. McKenley, P. B. Radha, T. R. Boehly, J. P. Knauer, F. J. Marshall, D. R. Harding, J. D. Kilkenny, D. D. Meyerhofer, T. C. Sangster, and R. L. McCrory, Phys. Plas. 11, 2763 (2004).

${ }^{20}$ E. I. Moses, IEEE Trans. Plas. Sc. 38, 684 (2010).

${ }^{21}$ R. T. Barton, Numerical Astrophysics, edited by J. M. Centrella, J. M. LeBlanc, R. L. Bowers, and J. A. Wheeler (Jones and Bartlett, Boston, 1985); R. E. Tipton, Megagauss 
Technology and Pulsed Power Applications, edited by C. M. Fowler, R. S. Caird, and D. J. Erickson (Plenum Press, New York, 1987).

${ }^{22}$ J. W. Miles, Report GAMD-7335, General Dynamics, 1966 (unpublished).

${ }^{23}$ D. C. Drucker, Mechanics Today, edited by S. Nemat-Nasser, Vol. 5 (Pergamon Press, New York, 1980).

${ }^{24}$ G. Terrones, J. Appl. Phys. 102, 034908 (2007).

${ }^{25}$ A. R. Piriz, J. J. L. Cela, and N. A. Tahir, Phys. Rev. E 80, 046305 (2009).

${ }^{26}$ A. L. Ortega, D. J. Hill, D. I. Pullin, and D. I. Meiron, Phys. Rev. E (in press).

${ }^{27}$ D. J. Steinberg, S. G. Cochran, and M. W. Guinan, J. Appl. Phys. 51, 1498 (1980). D. J. Steinberg, UCRL-MA-106439 (1996) (unpublished).

${ }^{28}$ D. J. Steinberg and C. M. Lund, J. Appl. Phys. 65, 1528 (1989).

${ }^{29}$ D. L. Preston, D. L. Tonks, and D. C. Wallace, J. Appl. Phys. 93, 211 (2003);F. J. Zerilli and R. W. Armstrong, J. Appl. Phys. 61, 1816 (1987).

${ }^{30}$ M. A. Monclus, T. J. Young, and D. Di Maio, J. Mat. Sc. 45, 3190 (2010).

${ }^{31}$ R. F. Cook, Science 328, 183 (2010).

${ }^{32}$ C. Lee, X. D. Wei, J. W. Kysar, and J. Hone, Science 321, 385 (2008).

${ }^{33}$ C. Cavailler, N. Fleurot, T. Lonjaret, and J. M. Di-Nicola, Plas. Phys. Contr. Fusion 46, B135 (2004).

${ }^{34}$ J. F. Barnes, D. H. Janney, R. K. London, K. A. Meyer, and D. H. Sharp, J. Appl. Phys. 51, 4678 (1980). 


\section{Figure Captions}

Fig. 1. Target and drive used in the paper. The target consists of an ablator (green), 75 $\mu \mathrm{m} \mathrm{CHBr} \mathrm{(12.5} \mathrm{atomic} \mathrm{percent} \mathrm{bromine)} \mathrm{followed} \mathrm{by} 25 \mu \mathrm{m}$ of $\mathrm{CH}$ (yellow), then 25 $\mu \mathrm{m}$ of Ta (violet), and $200 \mu \mathrm{m}$ of $\mathrm{CH}$. The RT pulse driving the target is the same as the early, 0-16 ns, part of the ignition pulse.

Fig. 2. The pressure in Megabars, the velocity in $\mu \mathrm{m} / \mathrm{ns}$, the density in $\mathrm{g} / \mathrm{cm}^{3}$, the melttemperature and the material temperature, both in $\mathrm{eV}$, of the front of the tantalum target driven by the RT pulse shown in Fig.1, all as functions of time in ns.

Fig. 3. The evolution of the peak-to-valley perturbations, initially $2 \mu \mathrm{m}\left(=2 \eta_{0}\right)$ at the $\mathrm{CH} / \mathrm{Ta}$ interface, as functions of time assuming 4 different strength properties for the tantalum: A: No strength; B: Steinberg-Guinan strength ${ }^{27}$; C: Steinberg-Lund $\operatorname{strength}^{28}$; and D: Modified Steinberg-Guinan strength with A (1.45/Mb originally) replaced by $2 \mathrm{~A}(2.9 / \mathrm{Mb})-$ See Appendix.

Fig. 4. Snapshots of the $\lambda=25 \mu \mathrm{m}, \eta_{0}=1 \mu \mathrm{m}$ simulation assuming the $\mathrm{SG}$ model for strength. The pseudo-color refers to the yield strength with a maximum of $90 \mathrm{~kb}$ (red). The evolution of the peak-to-valley amplitude is shown as curve B in Fig. 3.

Fig. 5. Three different methods of perturbing the $\mathrm{CH} / \mathrm{Ta}$ interface. Method $\mathrm{A}$ is commonly used with the metal (violet) and tamper (yellow) having identical perturbations. In Method B only the metal is perturbed, and in Method C only the tamper is perturbed.

Fig. 6. Same as Fig. 3 using Method C of Fig. 5 - The tantalum was initially flat and the tamper had $2 \eta_{0}=4 \mu \mathrm{m}$ peak-to-valley perturbations. 
Fig. 7. Same as Fig. 4 using Method $\mathrm{C}$ with $\lambda=25 \mu \mathrm{m}$ and $\eta_{0}=2 \mu \mathrm{m}$ in the $\mathrm{CH}$ only. The evolution of the peak-to-valley amplitude is shown as curve B in Fig. 6.

Fig. 8. Comparison of Methods A and C (Fig. 5), with and without SG strength, all at 20 ns. 


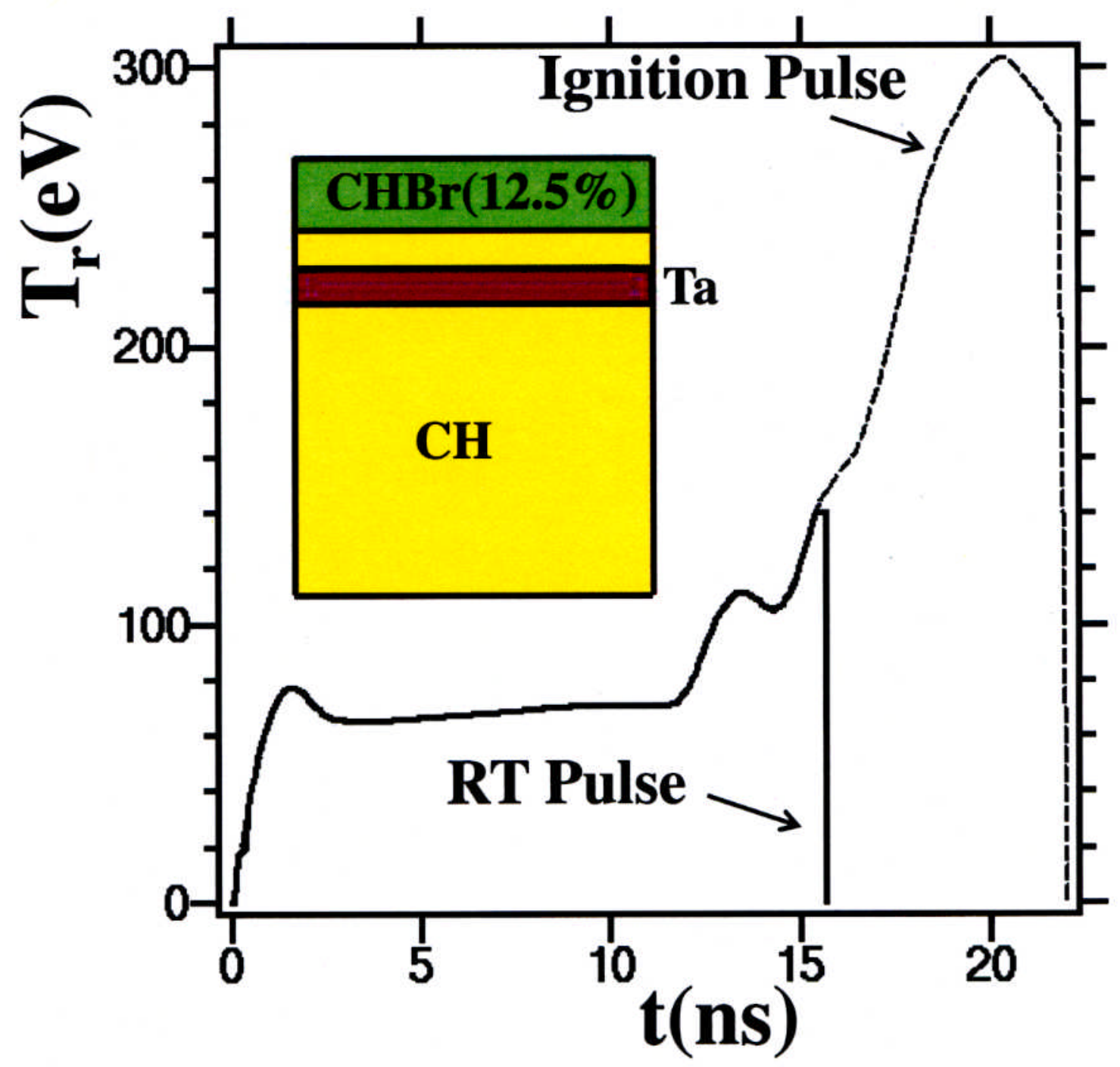

Fig. 1 


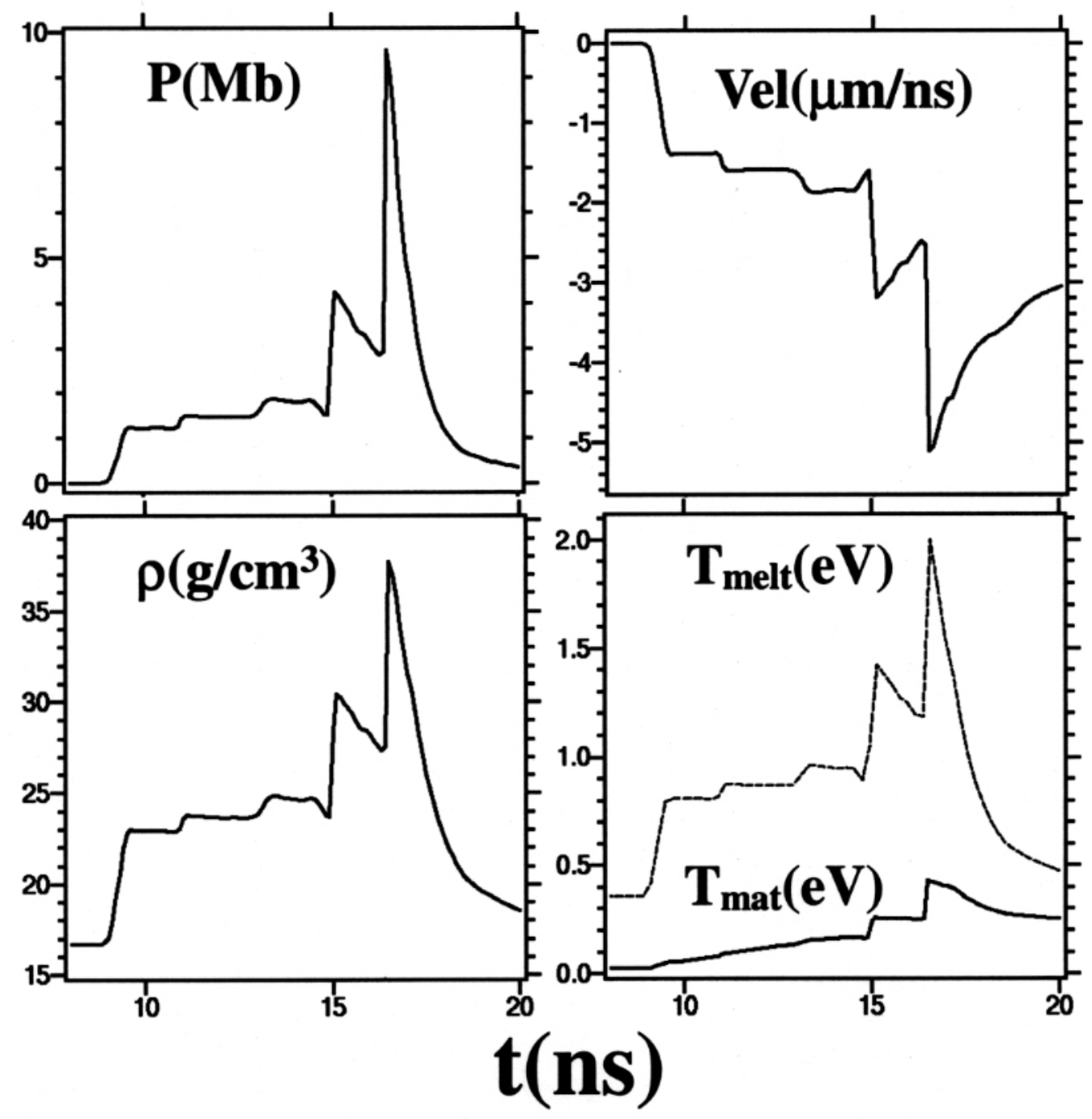

Fig. 2 


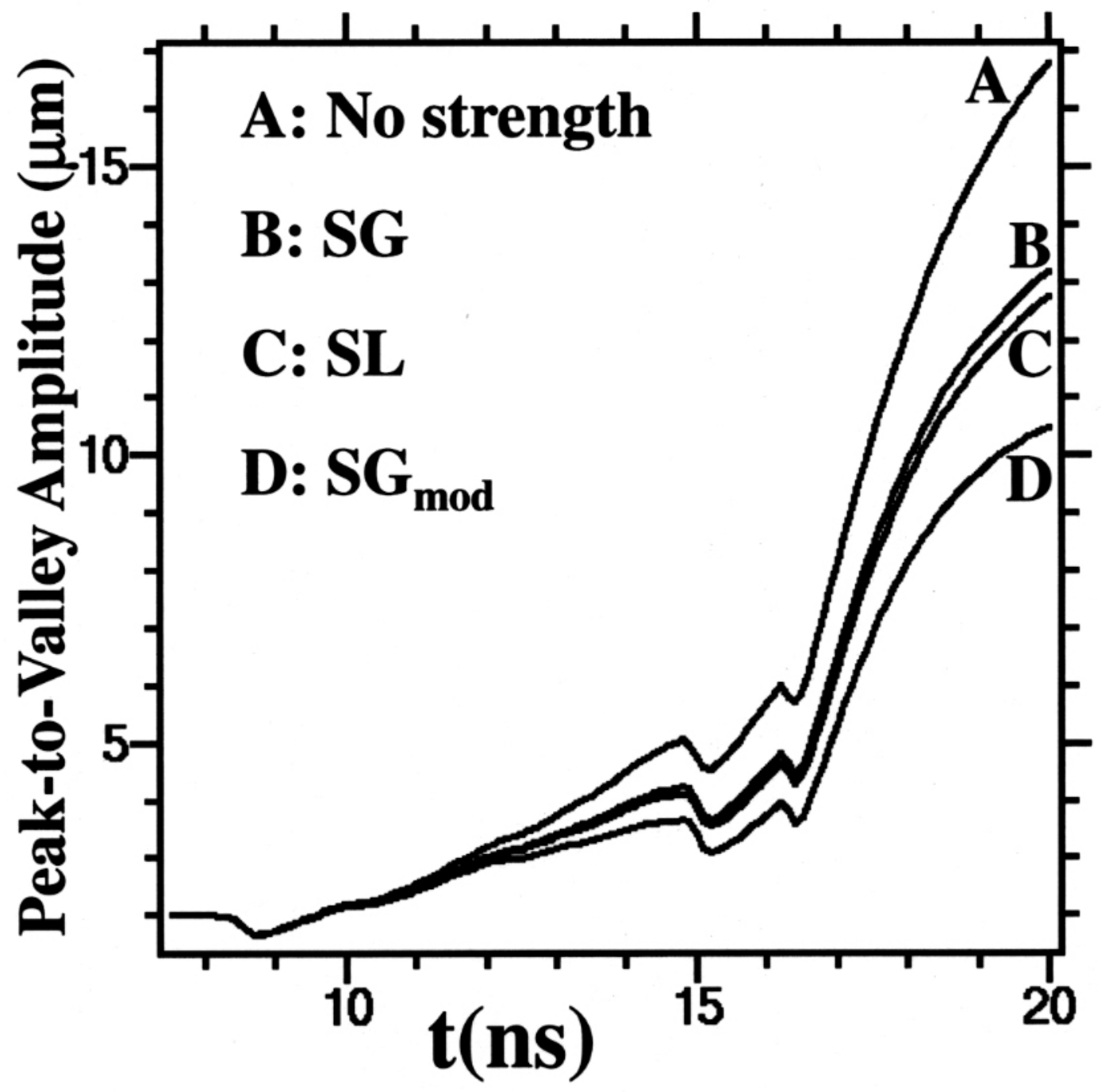

Fig. 3 

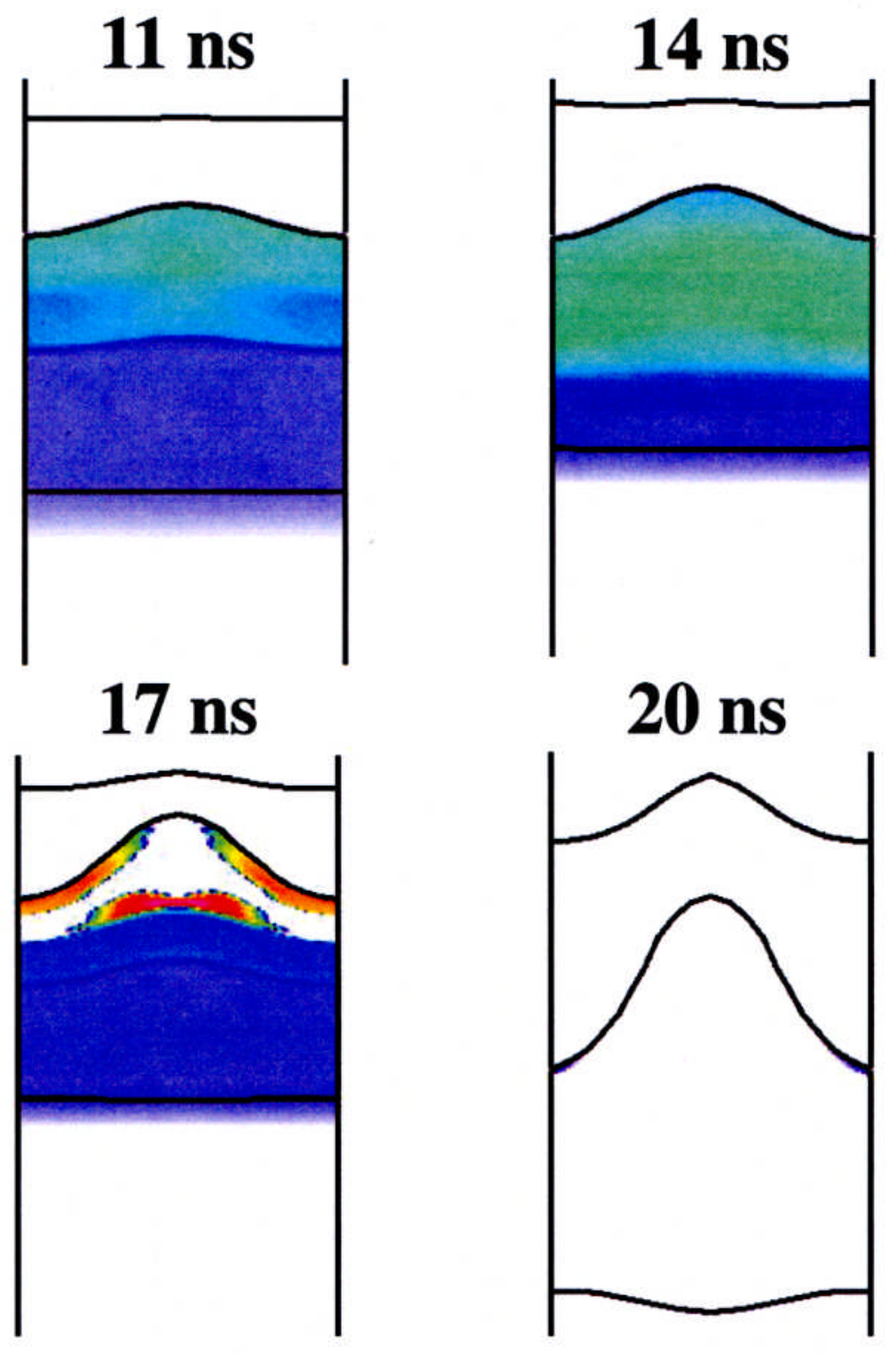

Fig. 4 


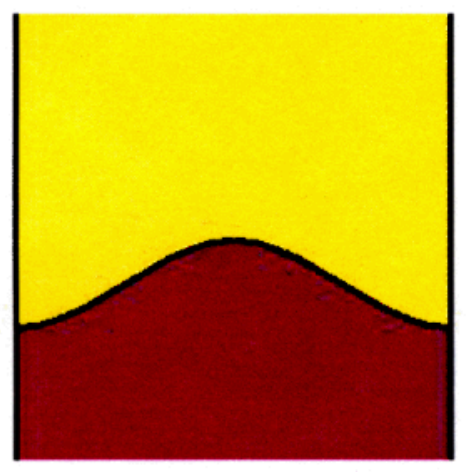

A

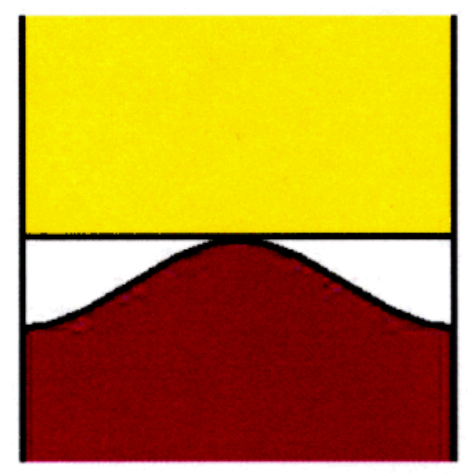

B

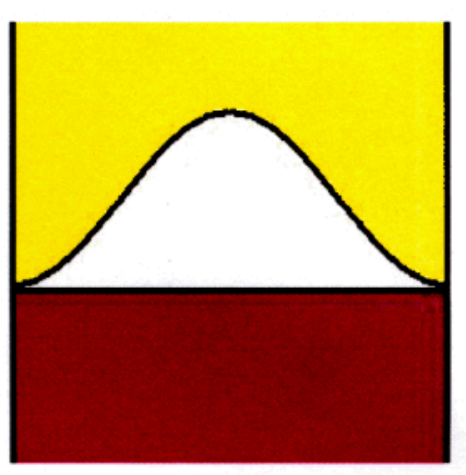

C

Fig. 5 


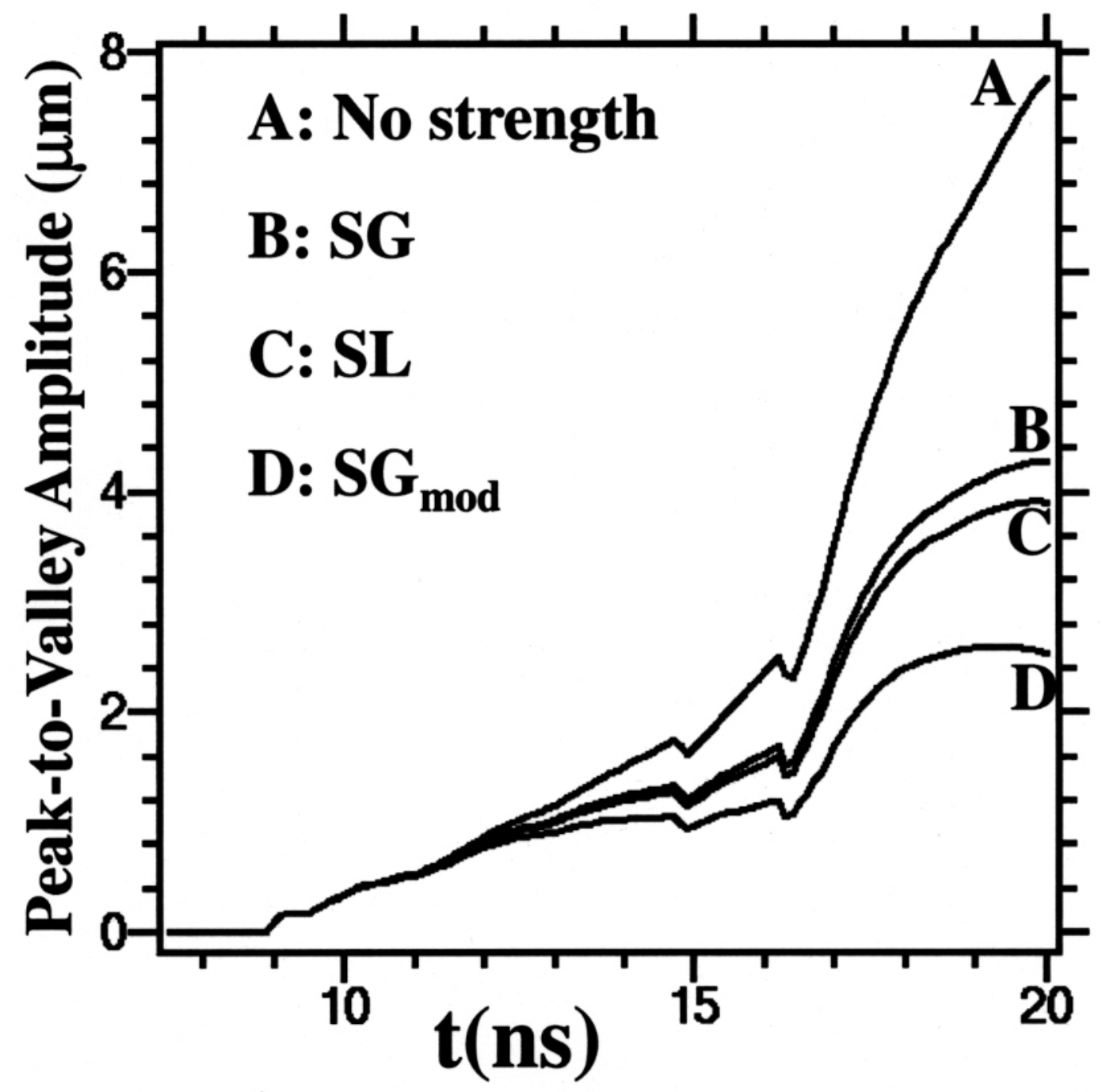

Fig. 6 
$11 \mathrm{~ns}$

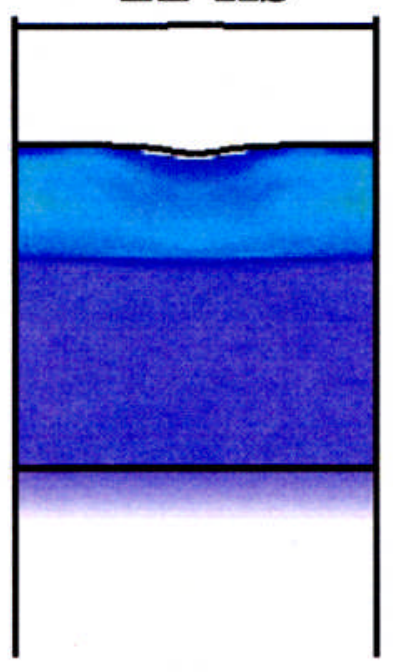

$17 \mathrm{~ns}$

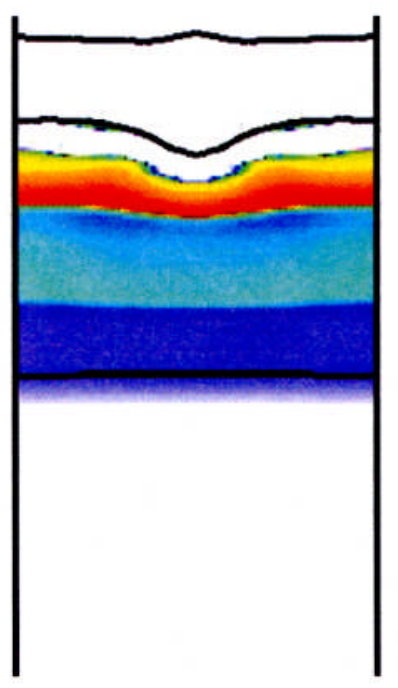

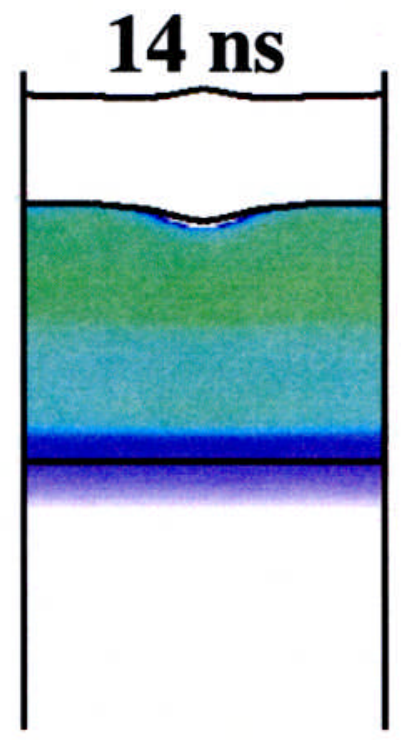

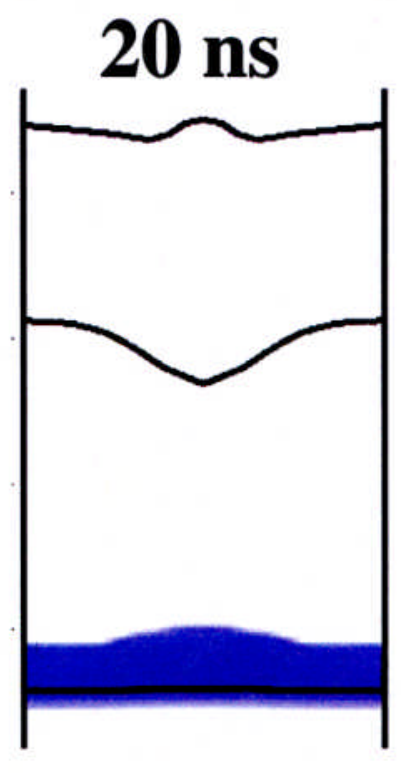

Fig. 7 


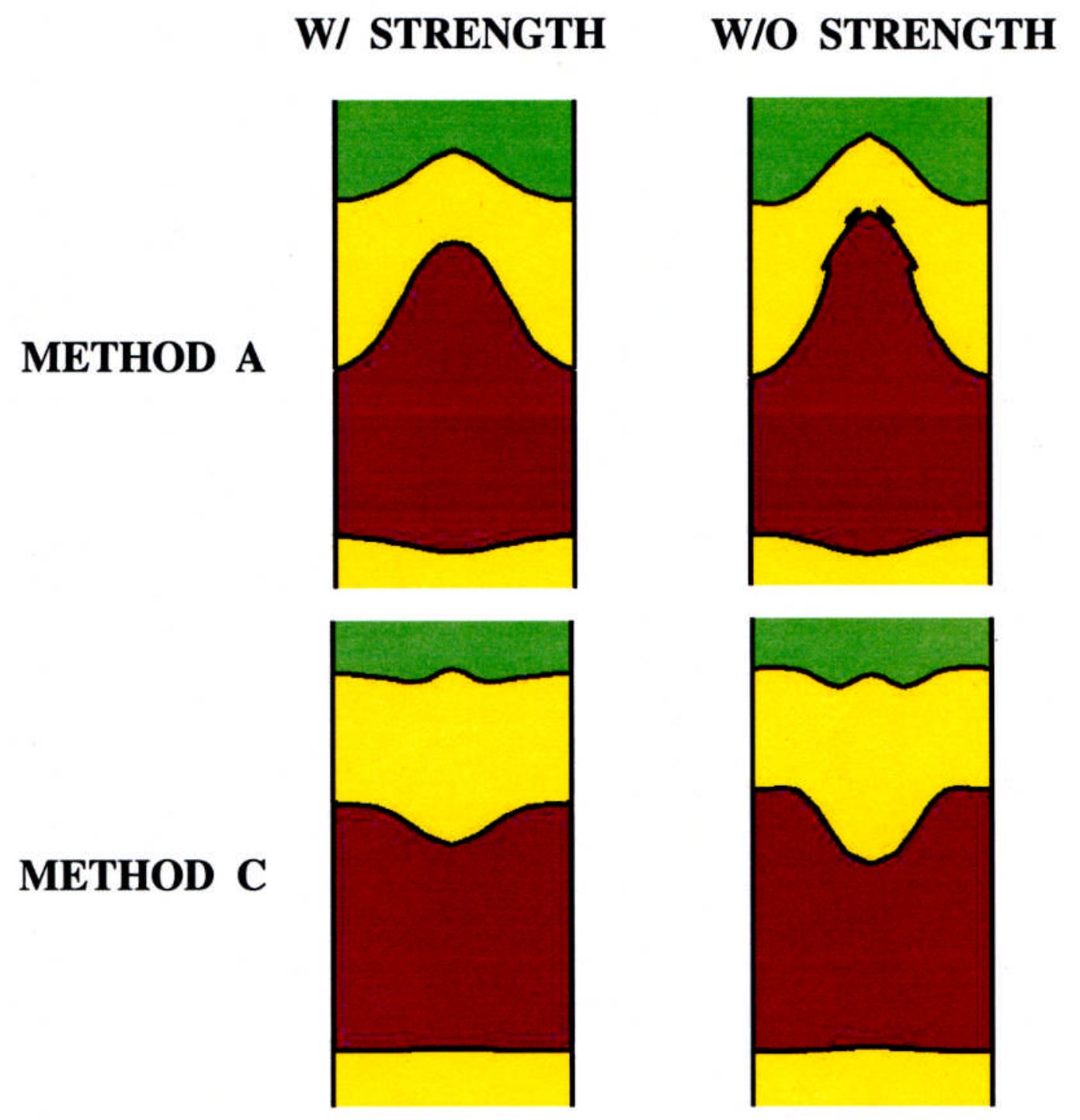

Fig. 8 